\title{
Study on Environmental Parameters and Wearing Electrocardiogram
}

\author{
Yang-Han Lee, ${ }^{1}$ Hsien-Wei Tseng, ${ }^{2 *}$ Yu-De Liao, ${ }^{1}$ \\ Ting-Wei Lin, ${ }^{1}$ Yi-Lun Chen, ${ }^{1}$ and Ying-Sen $\mathrm{Ho}^{1}$ \\ ${ }^{1}$ Department of Electrical and Computer Engineering, Tamkang University, Taiwan, R.O.C. \\ ${ }^{2}$ School of Mathematics and Information Engineering, Longyan University, Longyan, Fujian, China
}

(Received November 23, 2018; accepted February 15, 2019)

Keywords: IoT, cloud database, ECG, environmental sensing

Over the years, environmental changes have made people focus their attention on the quality of their surrounding areas and work environment. However, there are some dangerous substances in areas suitable for human survival and work. People often ignore harmful substances in the air (e.g., substances determined by temperature and humidity, carbon dioxide, and other harmful gases), resulting in physical problems. These substances are often too small to be observed and will severely jeopardize a human body that has been long fixed in the same place. Therefore, the purpose of this study is to assist humans in detecting the presence of hazardous substances in their living and work environments, and to improve such environments through sampling and inspection. Through this effort, humans can survive in a living and working space, and live and work in a comfortable environment. On the basis of the Arduino development edition, in this study, we transfer data to a cloud database through WiFi and combine the cloud database with the heart rate from an electrocardiogram to detect the human heart rate in the work environment to achieve $24 \mathrm{~h}$ real-time monitoring at home or in the workplace. By improving the flow field of the interior space, we can further reduce the indoor harmful substance level and optimize human life and work quality. For life and industrial hygiene safety consciousness awakening, in this study, we can promote human safety in the living and work environments by using a sensor to prevent potentially dangerous genetic lesions or derivatives of a disease. In addition, the scope of this study is not limited to the living space of people but extends to factories or other places with harmful substances. In a high-risk work environment, the equipment can be used to conduct risk preanalysis and detection in place of a human to achieve full assessment or environmental improvement, which eliminates the safety concerns of people in the environment.

\section{Introduction}

Over the years, environmental changes have made people focus their attention on the quality of their surrounding areas and work environment [Development of the core technology

*Corresponding author: e-mail: hsienwei.tseng@gmail.com

https://doi.org/10.18494/SAM.2019.2223 
of mobile-assist robots for the elderly health via Artificial Intelligence and Internet of Things (project number: MOST 107-2221-E-032-047-)]. However, there are some dangerous substances in areas suitable for human survival and work. People often ignore harmful substances in the air, resulting in physical problems. Therefore, we hope to achieve real-time monitoring of environmental parameters and study the correlation between the heart rhythm of subjects and environmental parameters by using a wearable electrocardiogram. ${ }^{(1-5)}$ The electrocardiogram is one of the most important indicators of heart health. It can achieve a certain diagnostic effect through a noninvasive method.

In a previous study, ${ }^{(6)}$ monitoring the use of wireless sensor network (WSN) technology to collect air quality data was proposed and the high potential of such technology was discussed. In the currernt study, we demostrated a monitoring and visualization approach to help big data play a role in the health and safety decisions of the shipping industry. We also identified other potential applications of WSN technology and the visualization of big data in the work environment, e.g., to monitor worker safety in high-risk industries and commodity quality in supply chain management.

In another previous study, ${ }^{(7)}$ the variation of electrocardiography (ECG)'s low frequency/ high frequency $(\mathrm{LF} / \mathrm{HF})$ ratio was proposed. As shown in Fig. 1, the sympathetic nervous system plays an important role in thermal discomfort. Once sympathetic excitation is achieved, temperature regulation disturbs the heat of the human body. Given the difference in LF/ HF ratio between the comfort and discomfort levels shown in Fig. 2, the LF/HF ratio can be considered as a physiological indicator of the thermal comfort level of the human body, as shown in Fig. 3.

Heart rate variability (HRV) is proposed in Ref. 8 as the index of thermal comfort in an office environment. Unlike existing mechanisms, this approach will allow people to predict the

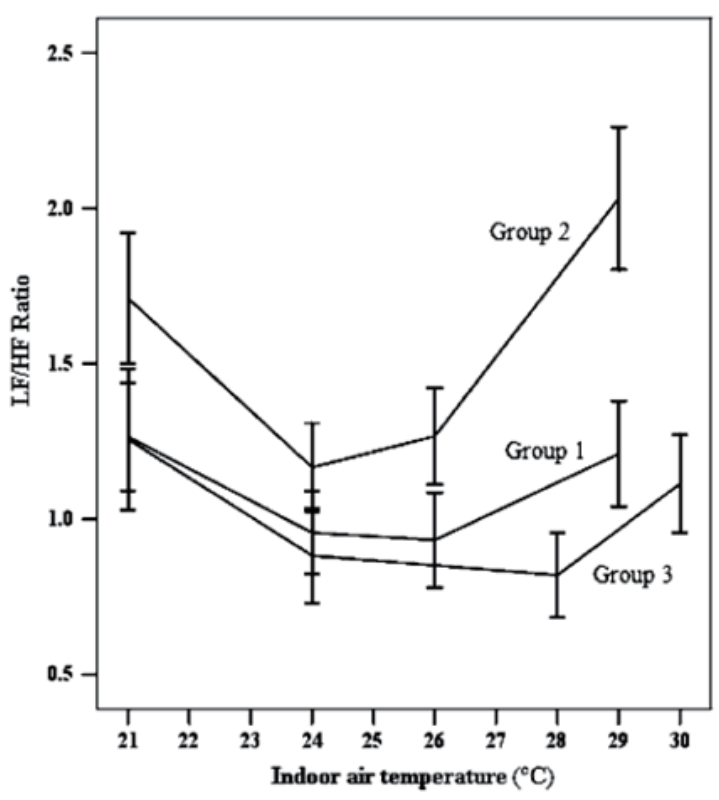

Fig. 1. Change in LF/HF ratio.

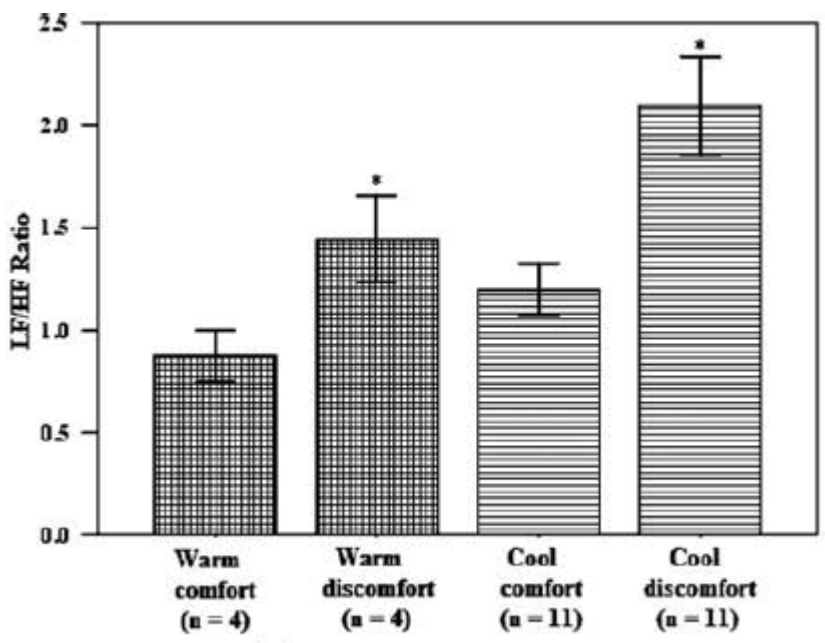

(a) Comfort and discomfort levels

Fig. 2. LF/HF ratio at comfort and discomfort levels. 


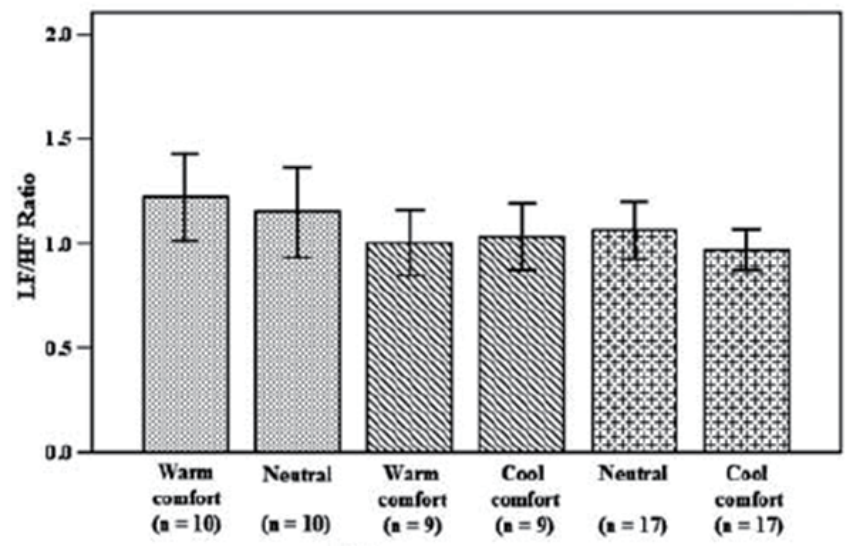

(b) Comfort levels

Fig. 3. EDR's LF/HF ratio at comfort level.

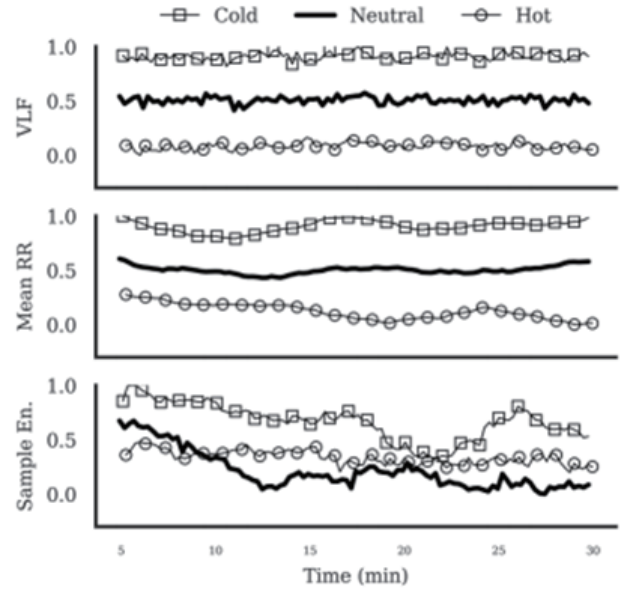

Fig. 4. Mean very low frequency power (VLF), mean R-R interval (RR), and HRV for all subjects.

physical response of thermal comfort to the surrounding heat, as shown in Fig. 4.

Most of the existing methods of environmental parameter detection are fixed-point measurement, and real-time data uploading cannot be achieved. Therefore, we hope to combine the cloud database with a web platform, so that viewers can view real-time data of environmental parameters anywhere and anytime. In this paper, real-time sensing of the parameters in the environment is proposed, and the arrhythmia analysis of wearing ECG is conducted for the personnel in the environment to observe the effect of the parameters in the environment on the heart rhythm of the subject. It will be confirmed by experiments that the heart rhythm of people changes with temperature, humidity, and carbon dioxide $\left(\mathrm{CO}_{2}\right)$ level, from which physiological information and physiological conditions of people in the environment can be observed. Thus, the method proposed here can be applied to various environments at home or in the workplace in the future for research and discussion. ${ }^{(9-14)}$

\section{Research Methods}

\subsection{Structure}

The structure used in this study is illustrated in Fig. 5. Arduino real-time measured data will be uploaded to the cloud, the WebCam camera will be employed for $24 \mathrm{~h}$ of real-time monitoring, the HRV of the subjects will be measured with a heartbeat band, and the above data will be displayed on a phone via Bluetooth to verify whether people in the environment feel physically ill from changes in the environment. On that basis, we can improve and achieve healthy environmental quality and maintain healthy living and work environments. ${ }^{(15-20)}$

\subsection{Introduction of hardware}

For the hard equipment used in this study, we employ an Arduino D1 development board combined with a DHT22 temperature and humidity module, an $\mathrm{MG} 811 \mathrm{CO}_{2}$ sensor 


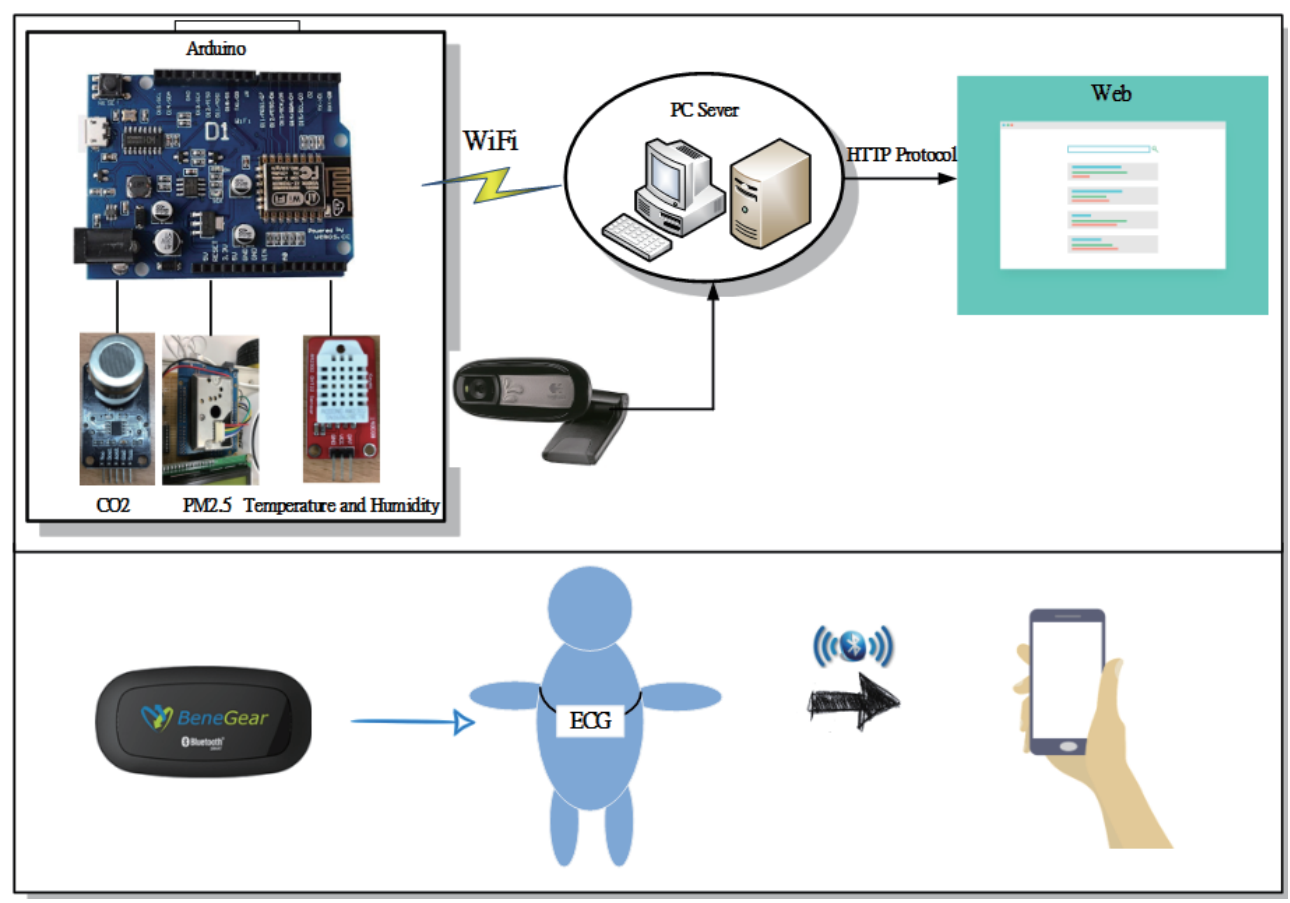

Fig. 5. (Color online) Structure.

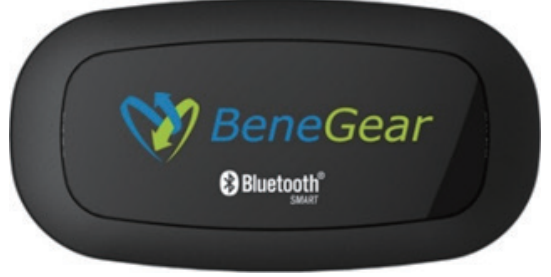

Fig. 6. (Color online) BeneGear Inc. ECG125.

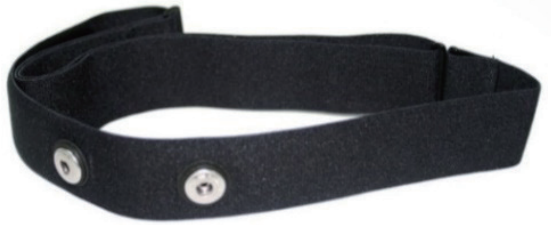

Fig. 7. (Color online) Heartbeat band.

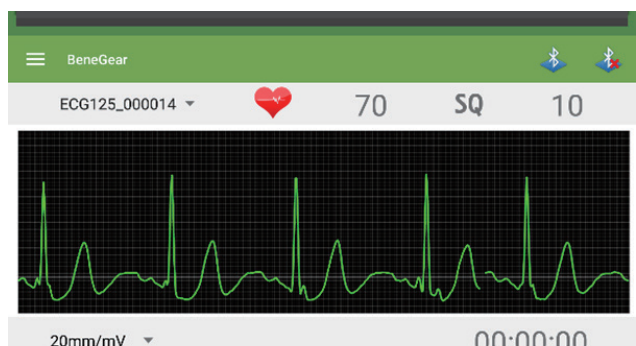

Fig. 8. (Color online) Observation of ECG signal mode based on Benegear Inc. HRM.

module, a PM2.5 dust sensing module, and an ESP8266 WiFi module to detect the uploaded environmental data. Subjects are required to wear the heartbeat equipment ECG125 (BeneGear Inc.) to obtain the ECG, as shown in Figs. 6-8. Logitech C107 WebCam is employed to present a webpage-based video. 


\subsection{Server architecture and web page presentation}

WampServer is a development environment for Windows Web consisting of Apache2, PHP, and MySQL database. PHP MyAdmin can be employed to manage the database, as shown in Fig. 9. PHP page syntax can be used to capture database data and display them on the web page, as shown in Fig. 10.

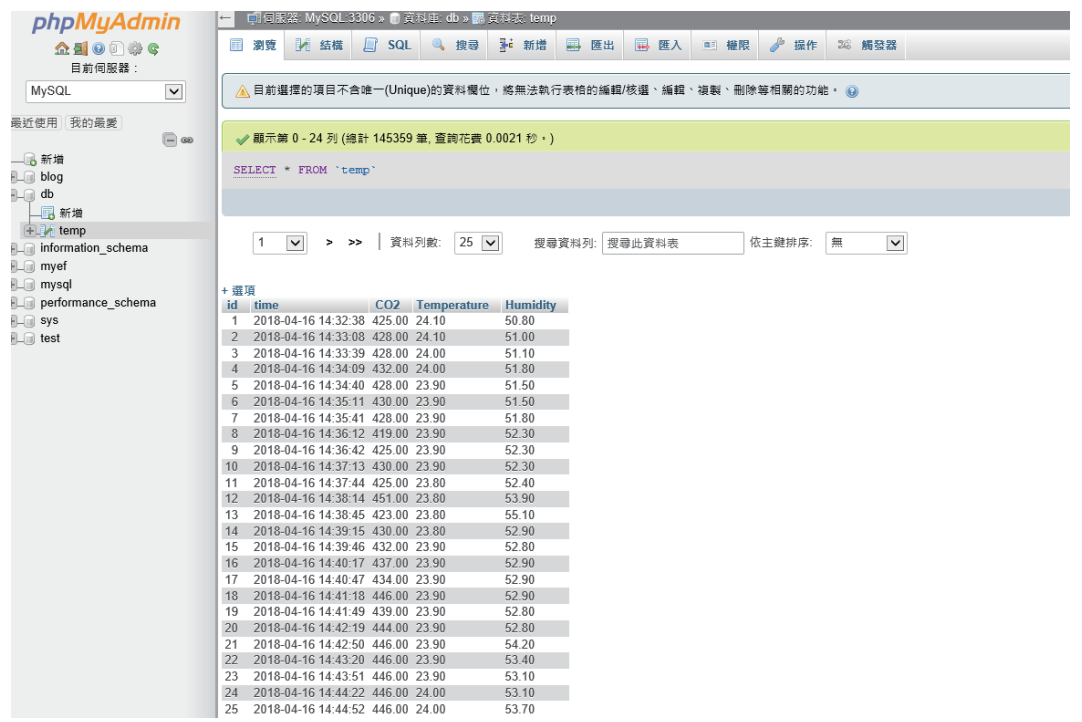

Fig. 9. (Color online) Cloud database.

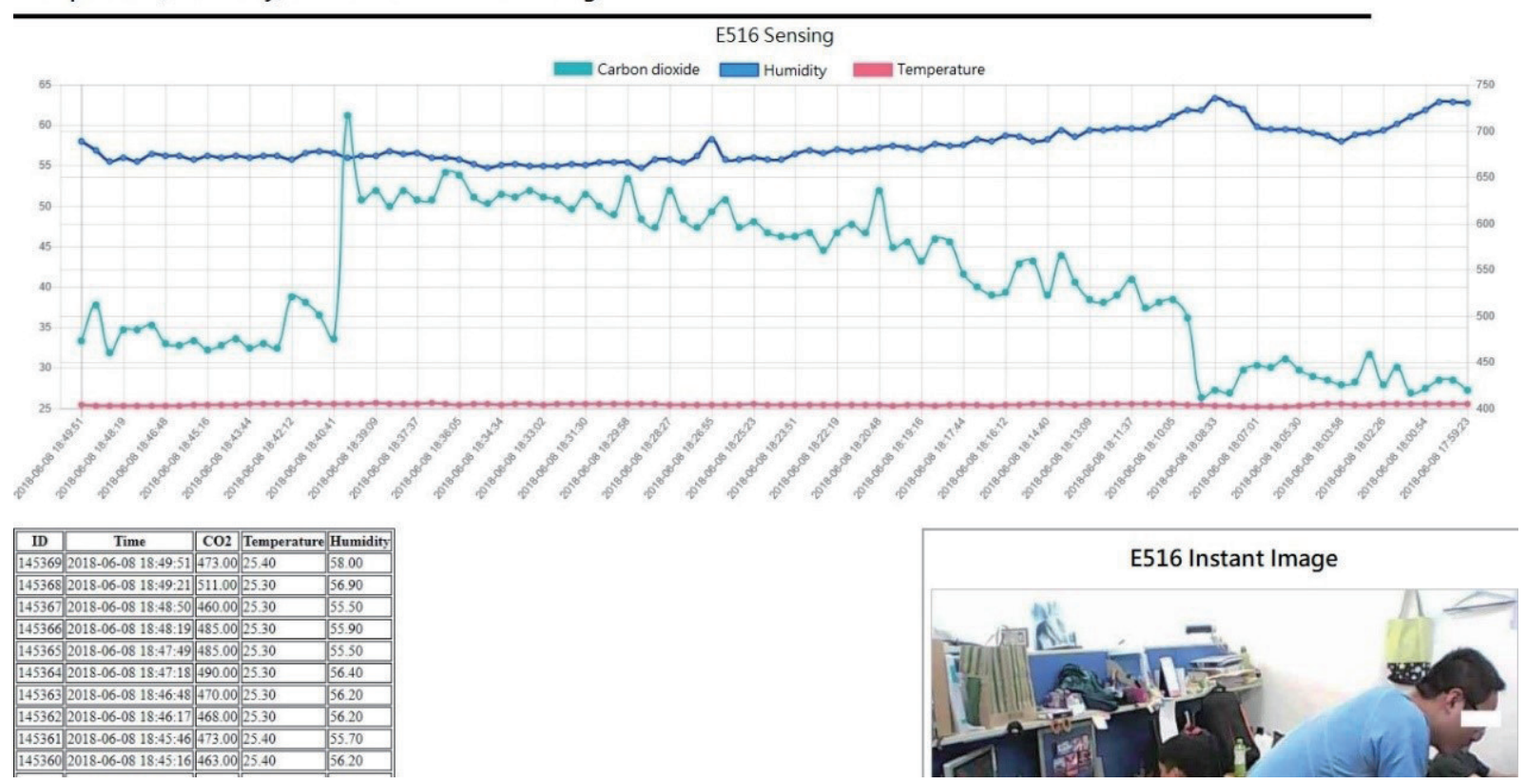

Fig. 10. (Color online) Web interface. 


\subsection{System flowchart}

The measurement is started. The camera, Arduino development platform, and heartbeat band are simultaneously turned on and the camera records the status of the experiment. The Arduino development platfrom measures the environmental data and uploads it to PHP. In addition, the ECG is measured through the heart band, and the ECG data is collected by a mobile phone. After collecting environmental and physiological data, the correlation between environmental quality and physical health is analyzed, as shown in Fig. 11.

\section{Results and Comparison}

\subsection{Correlation between PM2.5 and ECG}

As shown in Fig. 12, the subject was cooking in an airtight kitchen. The PM2.5 and ECG were measured, and then their correlation was analyzed. In the morning experiment that started at 00:05, the cooking time was 00:10, cooking was done at 00:30, and the measurement ended at 00:33. Figure 13 shows the relationship of HRV with temperature and humidity. Figure 14 shows the correlation between HRV and PM2.5 of the subject. Figure 15 shows subjects' ECG.

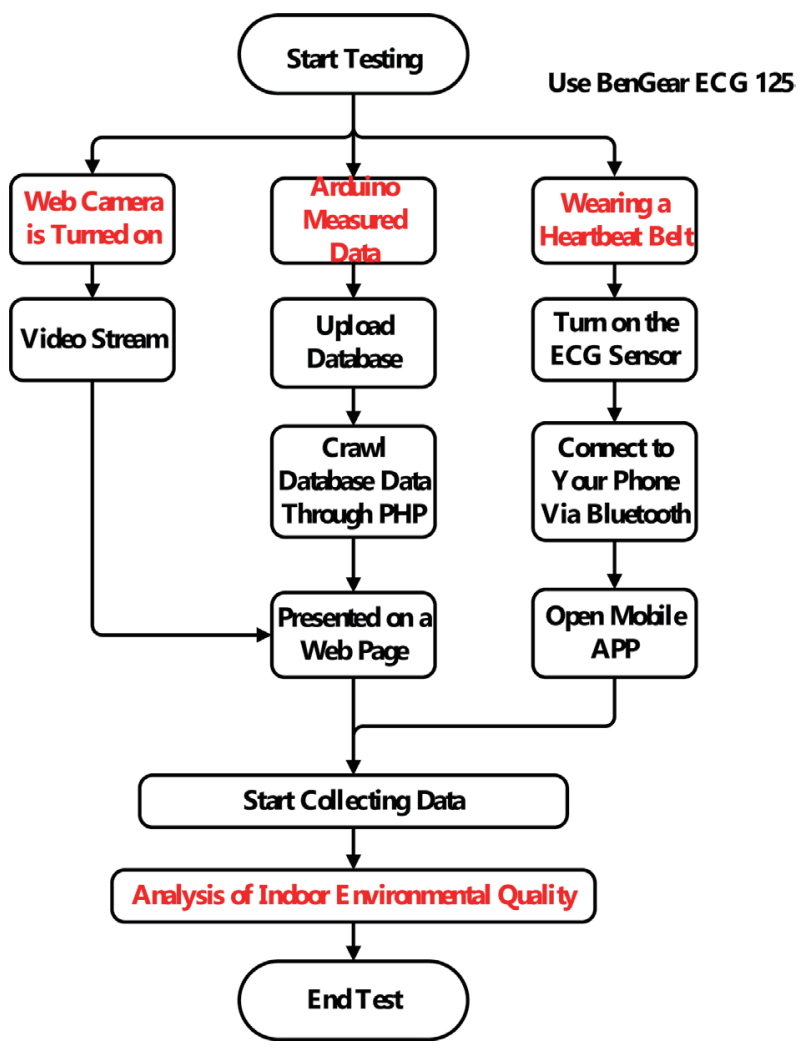

Fig. 11. (Color online) System flowchart. 


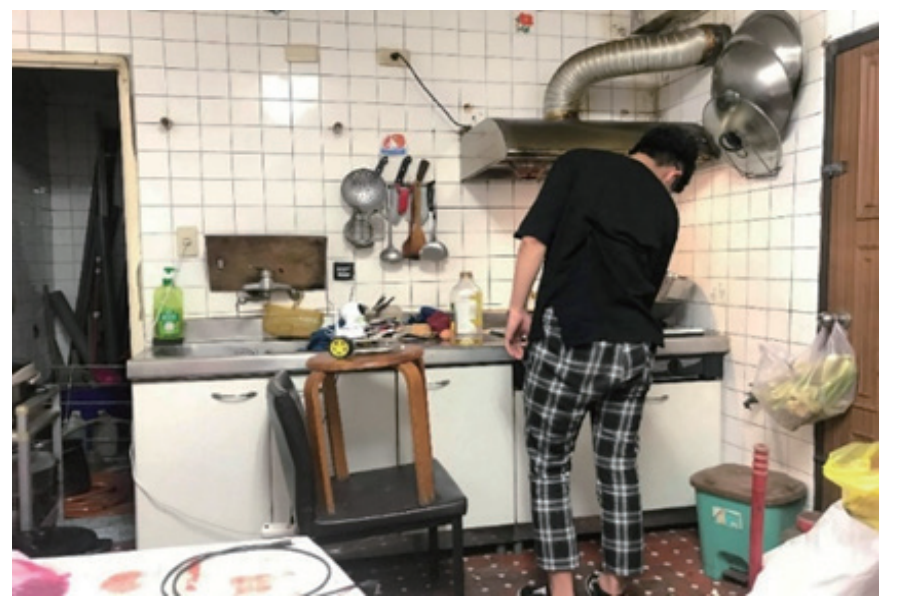

Fig. 12. (Color online) Test environment (kitchen).

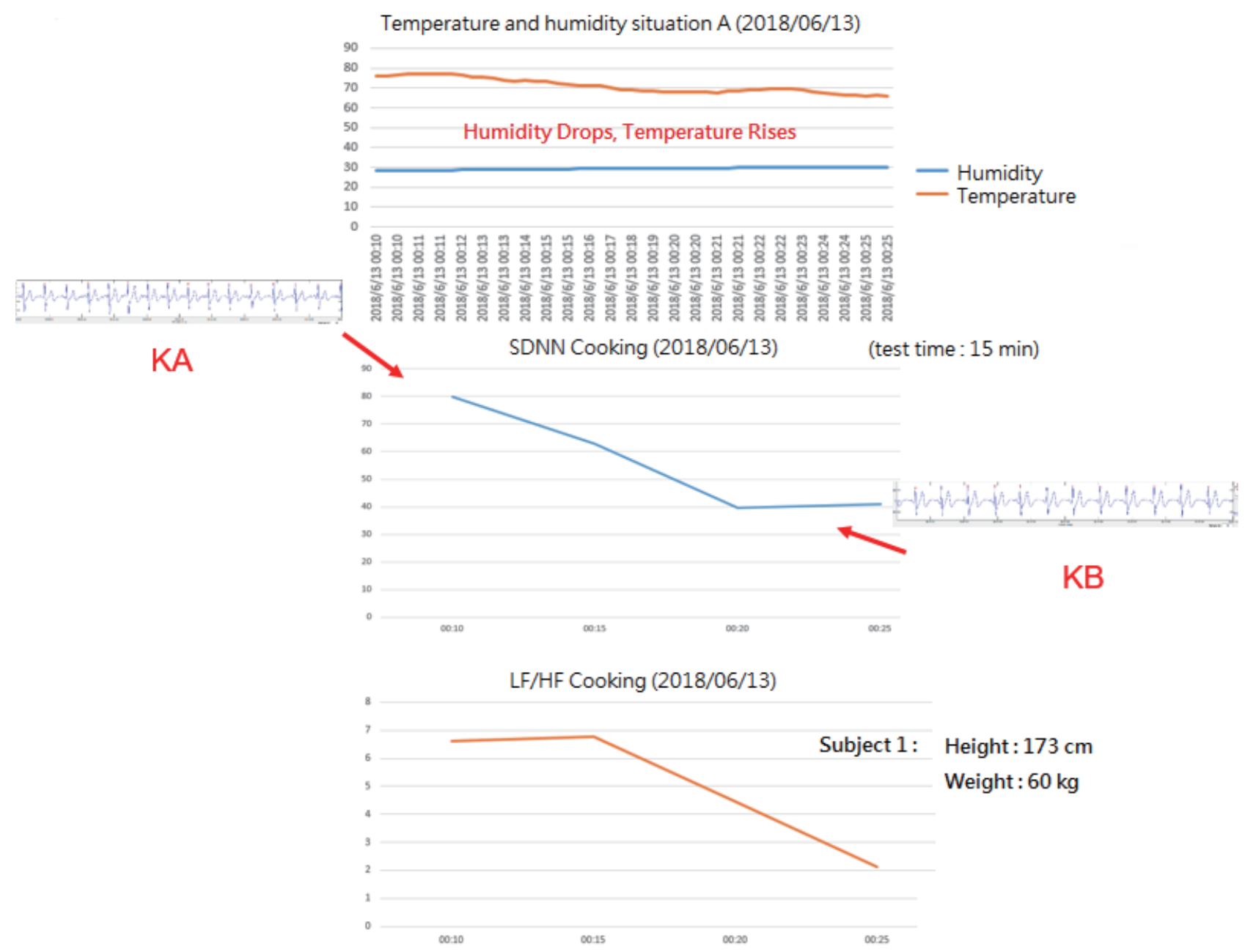

Fig. 13. (Color online) Relationship between HRV and temperature and humidity. 


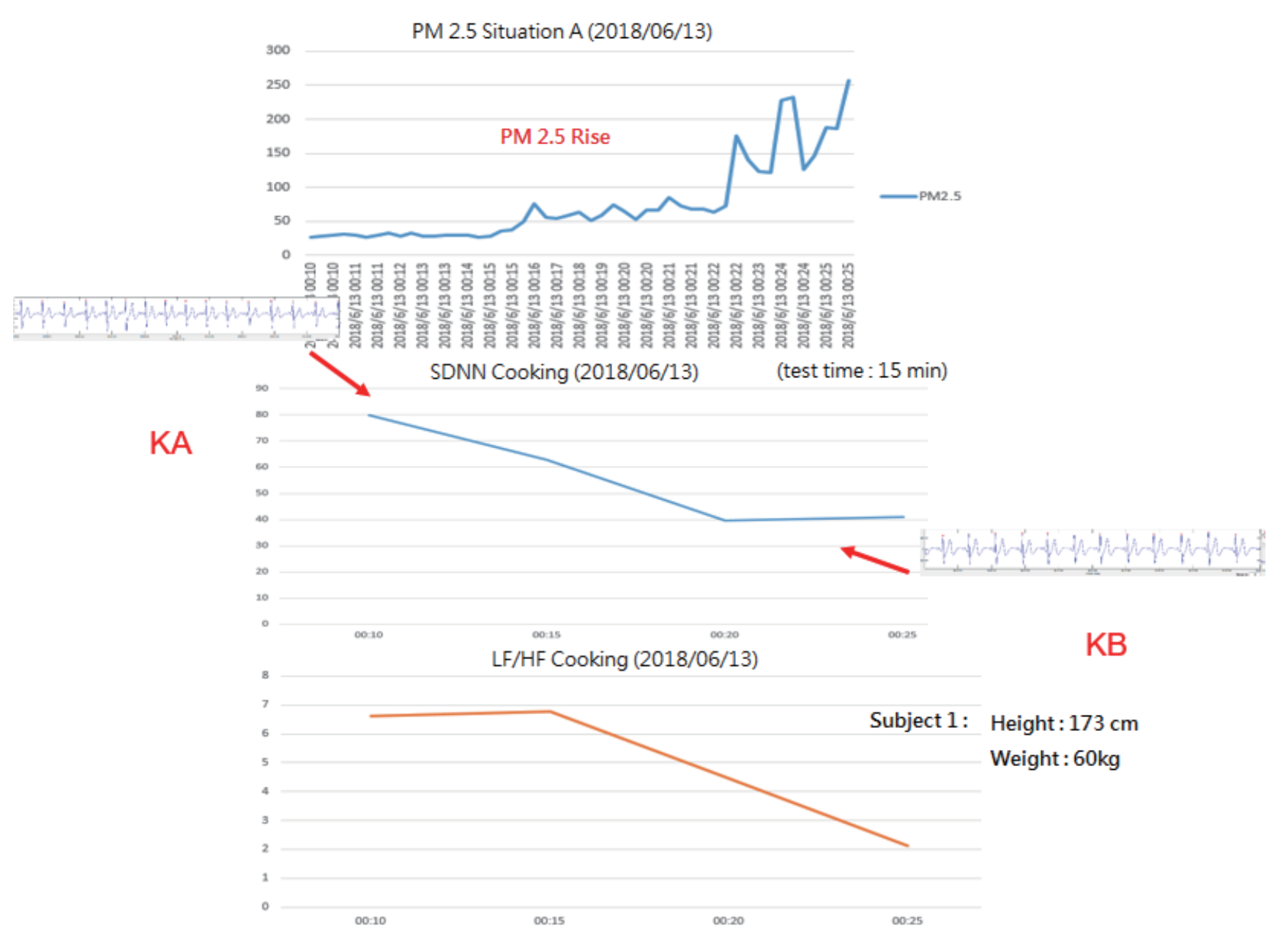

Fig. 14. (Color online) Correlation between HRV and PM2.5 of the subject.

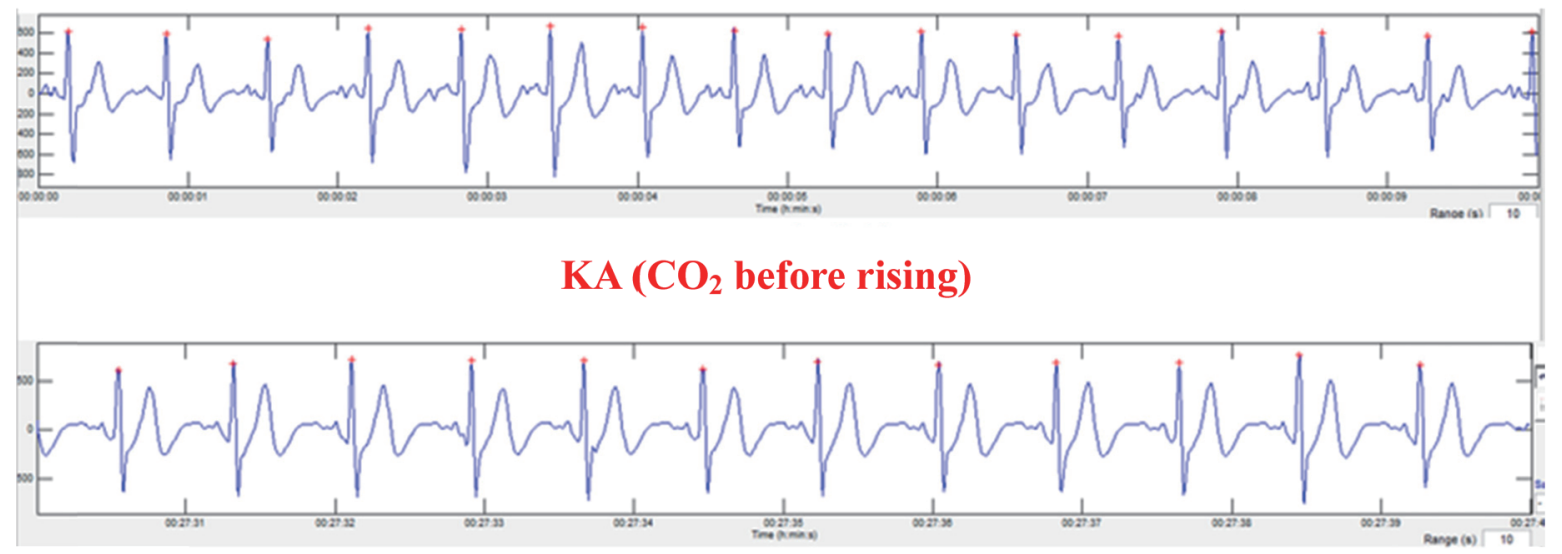

$\mathrm{KA}\left(\mathrm{CO}_{2}\right.$ after rising $)$

(Electrocardiogram takes 10s)

Fig. 15. (Color online) Subjects' ECG. 


\subsection{Correlation between $\mathrm{CO}_{2}$ and ECG}

As shown in Fig. 16, the subjects first measured $\mathrm{CO}_{2}$ and ECG in an airtight classroom. During the measurement, fans were added to improve air convection, as shown in Fig. 17. Finally, the correlation between $\mathrm{CO}_{2}$ and ECG was analyzed. The experimental test lasted from 15:42 to 17:50, the measurement was started at 15:42, and the half-time was 16:50. Fans were added to improve the indoor flow field. Then, the measurement was started at 17:10 and then ended at 17:50. A total of six subjects were included.

\section{Results of environmental classroom under test}

15:42 Measurement started

16:50 Half-time

17:10 Measurement restarted

(Improved indoor flow field)

17:50 Measurement ended

Figure 18 shows $\mathrm{CO}_{2}$ changes in classroom. Figure 19 shows the relationship of HRV with temperature and humidity of subject 1 . Figure 20 shows the relationship between $\mathrm{HRV}$ and $\mathrm{CO}_{2}$ of subjects 1 . Figure 21 shows the heart rate of subject 1 .

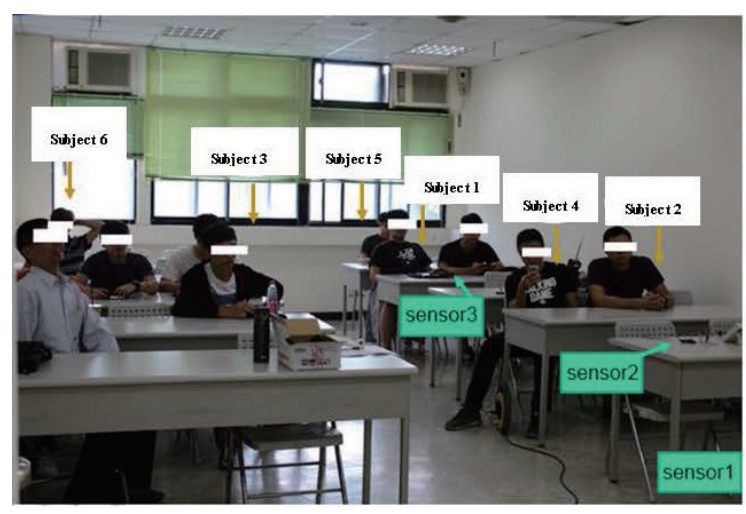

Fig. 16. (Color online) Measurement of classroom environment.

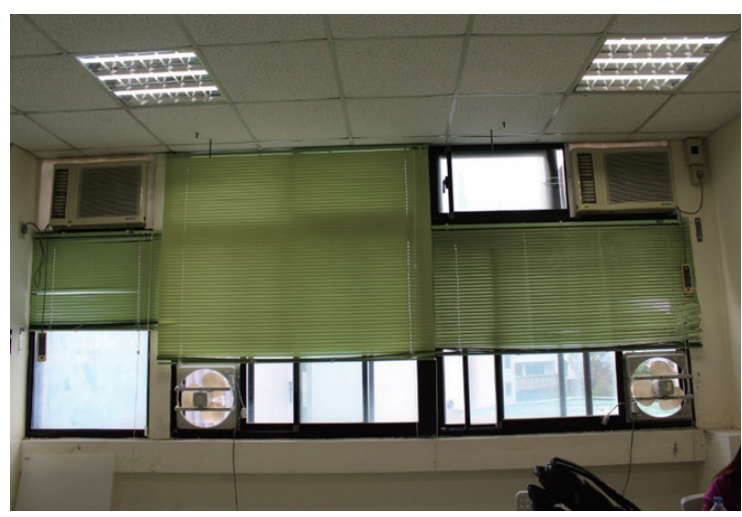

Fig. 17. (Color online) Locations of convection fans. 


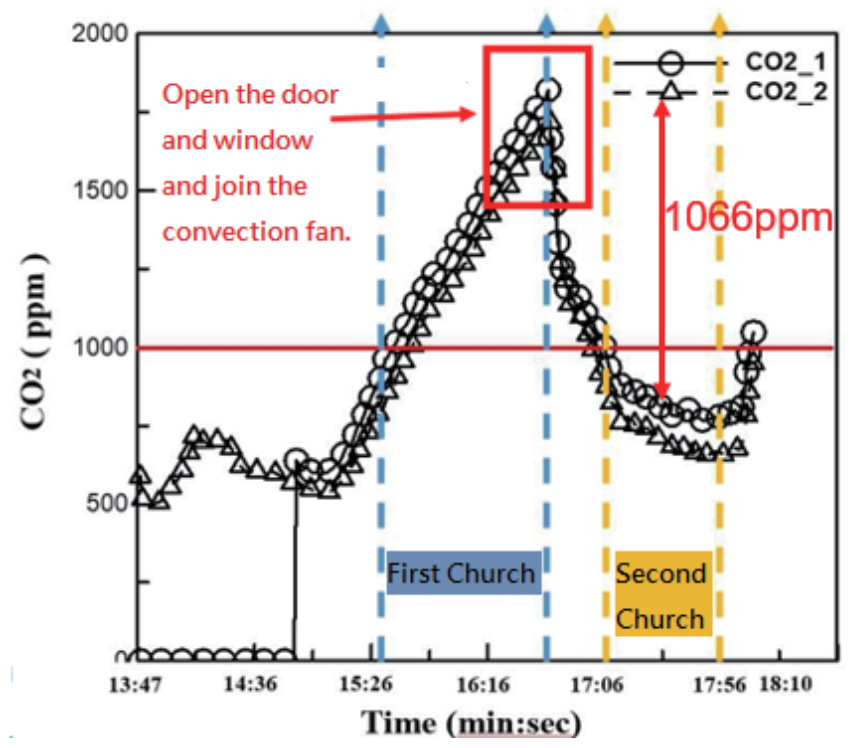

15:43 Start

16:41 Rest

17:02 Start

17:08 Dr. Su time

17:50 Dr. Su ended

Fig. 18. (Color online) $\mathrm{CO}_{2}$ changes in classroom.

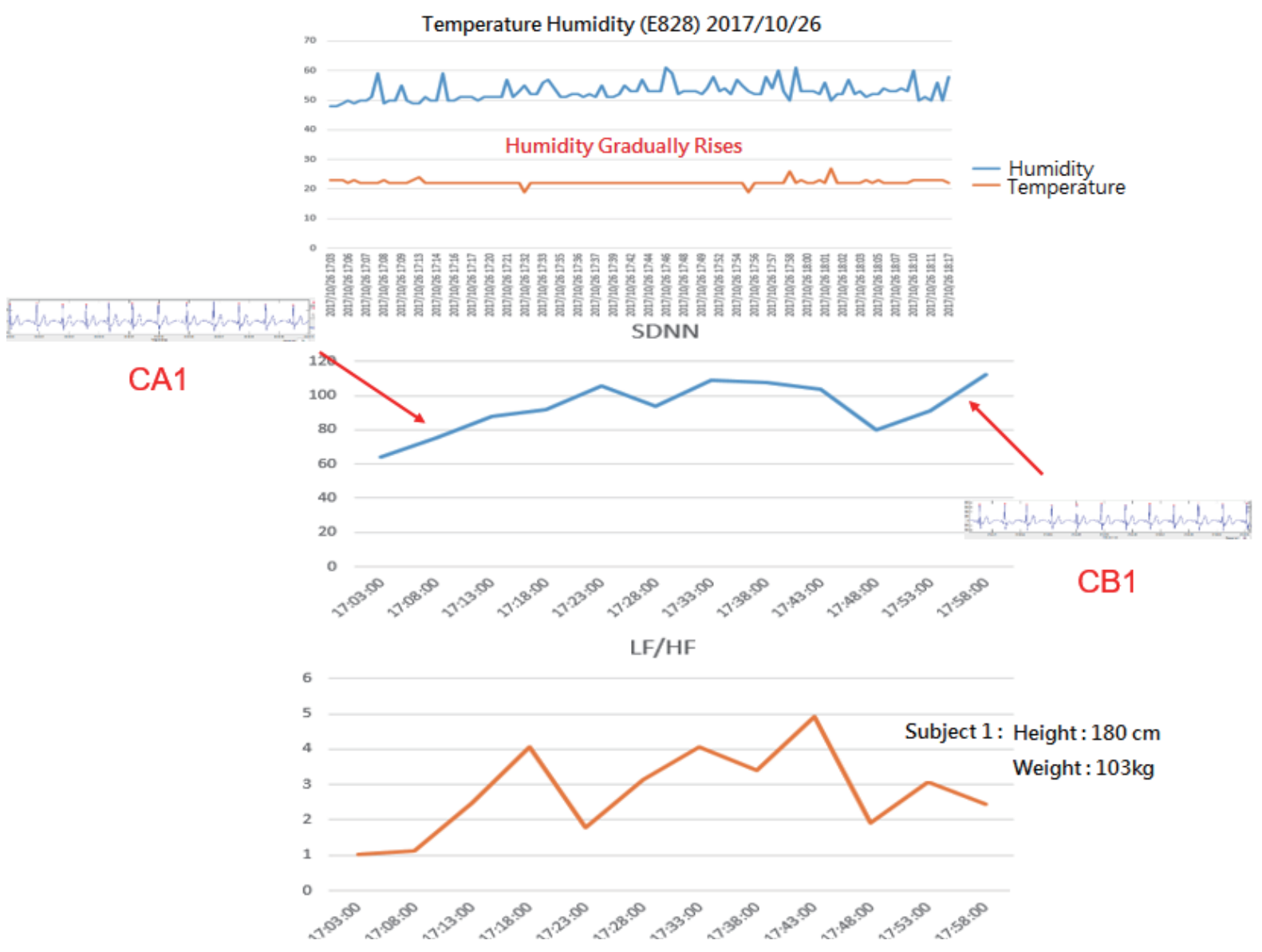

Fig. 19. (Color online) Relationship between HRV and temperature and humidity of subject 1. 


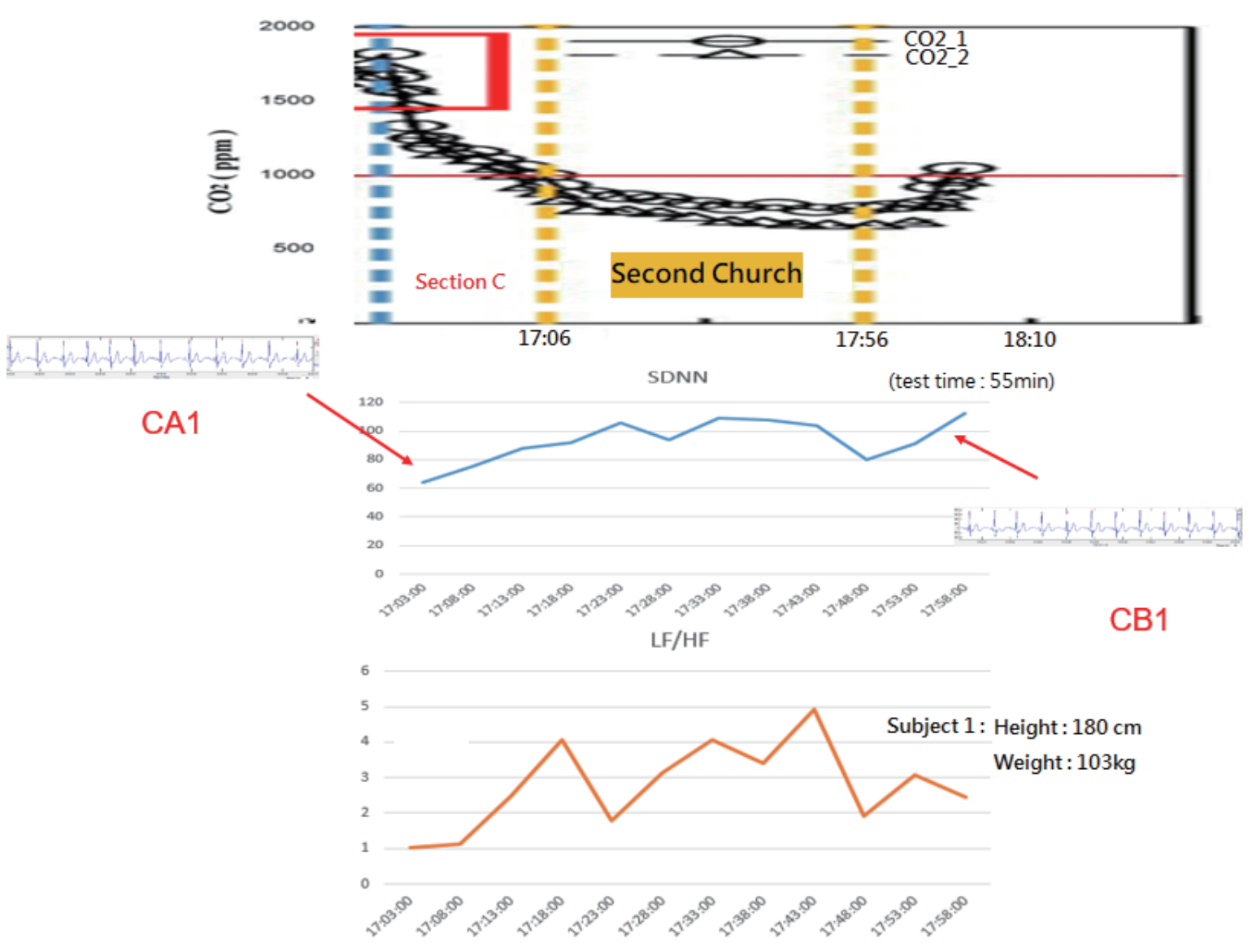

Fig. 20. (Color online) Relationship between $\mathrm{HRV}$ and $\mathrm{CO}_{2}$ of subject 1 .

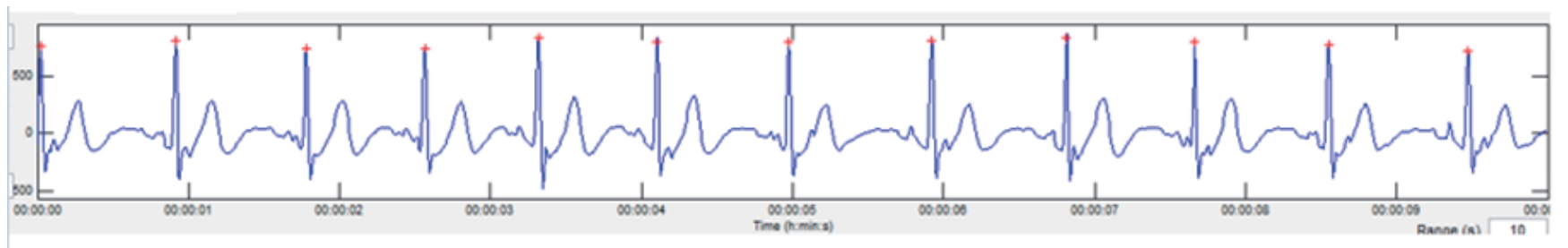

$\mathrm{CAl}\left(\mathrm{CO}_{2}\right.$ concentration is $\left.1800 \mathrm{ppm}\right)$

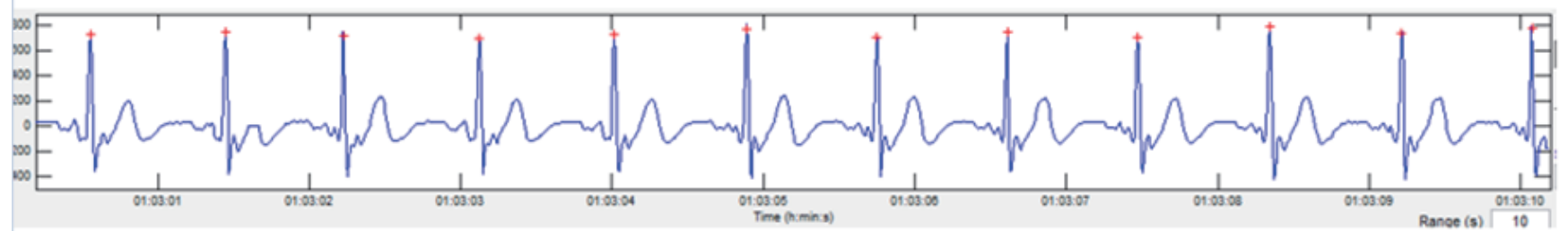

$\mathrm{CB} 2\left(\mathrm{CO}_{2}\right.$ after reduction in concentration)

Fig. 21. (Color online) Heart rate of subject 1. 


\section{Conclusion}

In this study, we primarily developed a web platform that is capable of detecting substances in the environment in real time and uploading them to a cloud database, allowing people to wear a load ECG device to compare the changes and determine the correlation between the substances in the environment and ECG. Many different situations were studied to compare the HRV analysis charts of the substances in air, and it was found that many changes indeed affect the heart rhythm of people.

The effects of $\mathrm{CO}_{2}$ and PM2.5 were found to be most obvious in many experiments. Some studies have also attempted to increase convection in air to reduce parameters that can affect the heart rhythm of the human body, and the electrocardiogram can sometimes slightly differ since people's behaviors might affect the changes in the electrocardiogram. Thus, these situations are also aspects to be further analyzed. The major contribution of this study is the introduction of an environmental sensor into a platform, introducing the real-time sensing of the human body to analyze the effects of environmental parameters on the human heart rhythm and achieving the convenience of real-time sensing using a wearable device.

\section{Acknowledgments}

This work was supported by a research project of the Ministry of Science and Technology, Republic of China, under grant MOST 107-2221-E-032-047.

\section{References}

1 G. S. Gupta and V. M. Quan: IEEE Sensors Applications Symp. (IEEE, 2018) 1.

2 J. Molka-Danielsen, P. Engelseth, V. Olešnaníková, P. Šarafín, and R. Žalman: IEEE 5th Int. Conf. Enterprise Systems (ES) (IEEE, 2017) 38.

3 W. Liu, Z. Lian, and Y. Liu: Eur. J. Appl. Physiol. 103 (2008) 361.

4 K. N. Nkurikiyeyezu, Y. Suzuki, Y. Tobe, G. F. Lopez, and K. Itao: IEEE 56th Annu. Conf. Society of Instrument and Control Engineers of Japan (IEEE, 2017) 1510.

5 A. R. Biswas and R. Giaffreda: IEEE 4th World Forum on Internet of Things (IEEE, 2014) 375.

6 Y. Feng, Y. Zhang, X. Cui, and X. Wang: IEEE Semicond. Technol. Int. Conf. (IEEE, 2018) 1.

7 D. Jarchi, D. Salvi, C. Velardo, A. Mahdi, L. Tarassenko, and D. A. Clifton: IEEE 15th Int. Conf. Wearable and Implantable Body Sensor Networks (IEEE, 2018) 144.

8 A. Malik and R. K. Sharma: IEEE Int. Conf. Trends in Electronics and Informatics (IEEE, 2017) 861.

9 S. C. Yener and R. Mutlu: IEEE Electric Electronics, Computer Science, Biomedical Engineerings' Meeting (IEEE, 2018) 1.

10 W. San-Um, C. Potiwanna, and S. Jakborvornphan: IEEE 5th Int. Conf. Business and Industrial Research (IEEE, 2018) 183.

11 S. T.Sanamdikar, S. T. Hamde, and V. G. Asutkar: IEEE Int. Conf. Energy, Communication, Data Analytics and Soft Computing (IEEE, 2017) 2407.

12 A. Smruthy and M. Suchetha: IEEE Sens. J. 17 (2017) 3092.

13 S. P. Awasarmol, S. Ashtekar, and A. Chintawar: IEEE Int. Conf. Energy, Communication, Data Analytics and Soft Computing (IEEE, 2017) 2850.

14 D. Morelli, L. Bartoloni, M. Colombo, D. Plans, and D. A. Clifton: Healthcare Technol. Lett. 5 (2017) 59.

15 D. Z. Wu, C. C. Sun, K. W. Chun, Y. X. Lin, and Y. H. Lin: IEEE/SICE Int. Symp. System Integration (IEEE, 2017) 493.

16 N. Özcan and M. Kuntalp: IEEE 10th Int. Conf. Electrical and Electronics Engineering (IEEE, 2017) 1385.

17 I. Tomasic, K. Khosraviani, P. Rosengren, M. Jörntén-Karlsson, and M. Lindén: 41st Int. Convention on Information and Communication Technology, Electronics and Microelectronics (IEEE, 2018) 330. 
18 M. M. Al Rahhal, Y. Bazia, H. AlHichri, N. Alajlan, F. Melgani, and R. R. Yager: Inf. Sci. 345 (2016) 340.

19 R. Wachter, K.Gröschel, G. Gelbrich, G. F. Hamann, P. Kermer, J. Liman, J. Seegers, K. Wasser, A. Schulte, F. Jürries, A. Messerschmid, N. Behnke, S. Gröschel, T. Uphaus, A. Grings, T. Ibis, S. Klimpe, M. Wagner-Heck, M. Arnold, E. Protsenko, P. U. Heuschmann, D. Conen, and M. Weber-Krüger: Lancet Neurol. 16 (2017) 282.

20 C. Orphanidou, T. Bonnici, P. Charlton, D. Clifton, D. Vallance, and L. Tarassenko: IEEE J. Biomed. Health Inf. 19 (2015) 832.

\section{About the Authors}

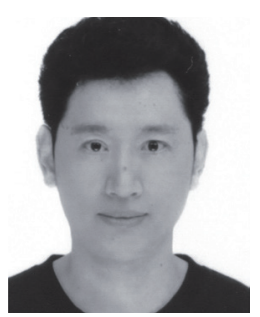

Yang-Han Lee received his B.S., M.S., and Ph.D. degrees in electrical engineering from National Taiwan University, Taipei, in 1987, 1989, and 1991, respectively. He joined the Department of Electrical Engineering, Tamkang University, Taipei, in 1994 and now serves as a professor. His main research interests include artificial intelligence, internet of things, and wireless communication systems.

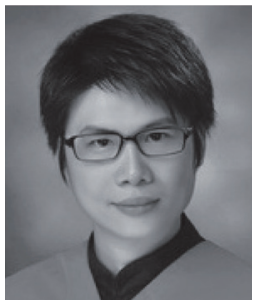

Hsien-Wei Tseng received his B.S. degree in electrical engineering from National Kaohsiung University of Applied Sciences in 2002 and his Ph.D. degree in electrical engineering from Tamkang University in 2010. He joined the School of Mathematics and Information Engineering, Longyan University, Longyan, Fujian, China, in Aug. 2017 and now serves as a professor. His main research interests include system design and performance evaluation in IoT and wireless communication systems.

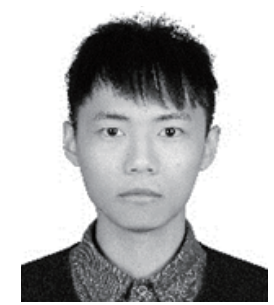

Yu-De Liao received his B.S. degree in computer and communication engineering in 2011 from DE LIN Institute of Technology and his M.S. degree in electronic engineering in 2013 from Tamkang University. He is currently a Ph.D. student at the Department of Electrical Engineering, Tamkang University. His main research interests include internet of things and wireless communication systems.

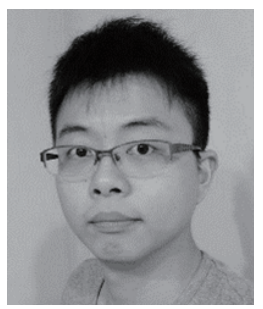

Ting-Wei Lin received his B.S. and M.S. degrees in Electrical Engineering from Tamkang University, Taipei, in 2013, and 2015, respectively. $\mathrm{He}$ is currently a Ph.D. student at the Department of Electrical and Computer Engineering, Tamkang University. His main research interests include artificial intelligence, internet of things, and embedded systems.

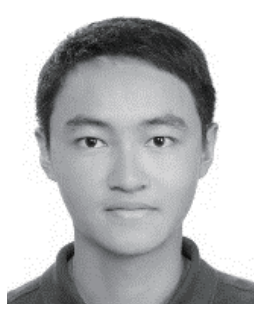

Yi-Lun Chen received his B.S. and M.S. degrees in electrical engineering from Tamkang University, Taipei, in 2015 and 2017, respectively. $\mathrm{He}$ is currently a Ph.D student at the Department of Electrical and Computer Engineering, Tamkang University. His main research interests include artificial intelligence, internet of things, and embedded systems. 
Ying-Sen Ho received his B.S. and M.S. degrees in electrical engineering from Tamkang University, Taipei, in 2009 and 2011, respectively. $\mathrm{He}$ is currently a Ph.D. student at the Department of Electrical and Computer Engineering, Tamkang University. His main research interests include internet of things and wireless communication systems. 